\title{
Long-lasting Molecular Orientation Induced by a Single Terahertz Pulse
}

\author{
Long $\mathrm{Xu},{ }^{*}$ Ilia Tutunnikov, ${ }^{*}$ Erez Gershnabel, Yehiam Prior, and Ilya Sh. Averbukh ${ }^{\dagger}$ \\ AMOS and Department of Chemical and Biological Physics, \\ The Weizmann Institute of Science, Rehovot 7610001, Israel
}

\begin{abstract}
Control of molecular rotations by laser fields is an active area of research focusing on the alignment/orientation of otherwise isotropic molecular samples. Oriented molecules are useful in many applications, such as molecular orbital tomography, femtosecond imaging of molecular structure, dynamics and chemical reaction control. In this work, we present a novel, previously unreported phenomenon appearing in thermal ensembles of non-linear polar molecules excited by a single $\mathrm{THz}$ pulse. We find that the induced orientation lasts long after the excitation pulse is over. In the case of symmetric-top molecules, the ensemble averaged orientation remains indefinitely constant after the pulse, whereas in the case of asymmetric-top molecules the orientation persists for a long time after the end of the pulse. We discuss the underlying mechanism, study its non-monotonous temperature dependence, and show that there exists an optimal temperature for the maximal residual orientation. The persistent orientation implies long lasting macroscopic dipole moment, which may be probed by second (or higher order) harmonic generation.
\end{abstract}

Introduction.- Molecular alignment and orientation, mostly by ultrashort optical pulses, have attracted considerable interest owing to their extreme importance in a variety of applications in physics, chemical reaction dynamics, and attosecond electron dynamics (for reviews see, e.g. [1-5] and references therein). Independently, rapid advances in terahertz $(\mathrm{THz})$ technology introduced high energy tabletop sources of ultrashort THz pulses [6]. In recent years, $\mathrm{THz}$ pulses were utilized to orient polar linear molecules [7-9], and later on, symmetric- [10] and asymmetric-top molecules [11]. The pioneering theoretical studies of the hybrid route to orientation, combining $\mathrm{THz}$ and optical pulses [12-14], were followed by extended theoretical research [15-18] inspired by the recent development of the $\mathrm{THz}$ technology, and finally resulted in an experimental demonstration of enhanced orientation [9]. The free motion of symmetric- and asymmetrictops is a standard textbook, classical and quantum mechanical topic, but the addition of interaction with an external field complicates the problem considerably, with just a few known integrable special cases (see [19, 20] and references therein). When a linear polar molecules is excited by a $\mathrm{THz}$ pulse, the induced orientation shows a short transient signal riding on zero baseline, with periodic recurrences (quantum revivals [21-23]), while keeping the time averaged signal zero. Clearly, the alignment and orientation of thermal molecular gases require ensemble averaging, bringing yet another level of complexity.

In this work, we theoretically study, classically and quantum-mechanically, the orientation of a rarified gas of symmetric- and asymmetric-top molecules excited by a single linearly polarized $\mathrm{THz}$ pulse. We demonstrate that the $\mathrm{THz}$ pulse not only induces a short time transient dipole signal (transient orientation), but also results in a steady molecular orientation that persists for a long time after the end of the pulse. In the case of symmetric-top molecules, the ensemble-averaged long-time orientation remains constant, while for asymmetric-top molecules, it persists on a time scale exceeding the duration of the pulse by several orders of magnitude. This behaviour is characteristic for complex molecules, and it is absent in the case of commonly studied linear molecules. We show that this phenomenon is classical in nature and its underlying mechanism is revealed and discussed. The degree of orientation has a non-monotonous temperature dependence, which is demonstrated and qualitatively explained.

Equations of motion.-The Hamiltonian describing molecular rotation driven by external time-dependent field interacting with the molecular dipole is given by [24] $H(t)=H_{R}+H_{\text {int }}(t)$, where $H_{R}$ is the rotational kinetic energy Hamiltonian and $H_{\text {int }}(t)=-\boldsymbol{\mu} \cdot \mathbf{E}(t)$ is the molecular dipole-field interaction. The electric field of the THz pulse is modeled as $\mathbf{E}(t)=E_{0}\left(1-2 \kappa t^{2}\right) e^{-\kappa t^{2}} \mathbf{e}_{Z}$ [25]. Here $E_{0}$ is the peak amplitude, $\kappa$ determines the width of the pulse, and $\mathbf{e}_{Z}$ is the unit vector along the laboratory $Z$ axis. Note that the time integral of the electric field is zero. Figure 1 shows the shape of the field, for parameters typical for currently available $\mathrm{THz}$ pulses [26-29]. Here, we consider the problem both classically and quantum mechanically. For the quantum mechanical treatment, the wave function is expanded in the basis of free symmetric-top wave functions $|J K M\rangle$ [30]. Here $J$ is the total angular momentum, while $K$ and $M$ denote its projections on the molecule-fixed axis and the laboratory-fixed $Z$ axis, respectively. The timedependent Schrdinger equation $i \hbar \partial_{t}|\Psi(t)\rangle=H|\Psi(t)\rangle$ is solved by numerical exponentiation of the Hamiltonian matrix (see Expokit [31]). We average the orientation signal over the initial thermal state of the molecular rotor. In the case of symmetric-top molecules, the nuclear spin statistics [32] is taken into account. A detailed description of our scheme is presented in Supplementary Note 1.

In the classical limit, the rotational motion of rigid 


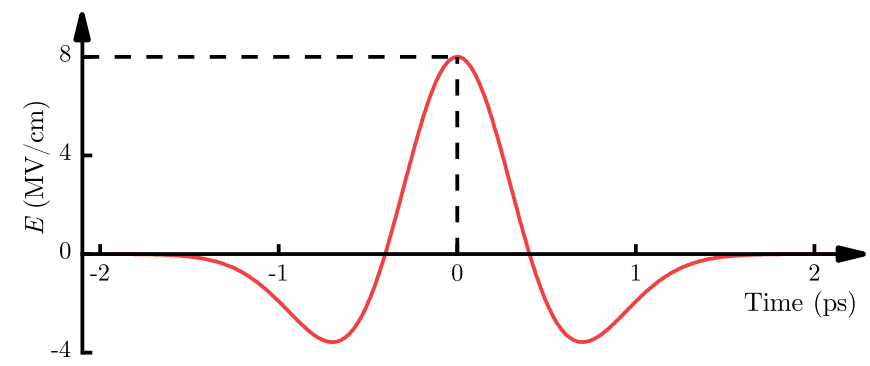

Figure 1. Time-dependent field amplitude of the $\mathrm{THz}$ pulse defined by $E(t)=E_{0}\left(1-2 \kappa t^{2}\right) e^{-\kappa t^{2}}$, where $E_{0}=8.0 \mathrm{MV} / \mathrm{cm}$ and $\kappa=3.06 \mathrm{ps}^{-2}$. Note that the time integral of the electric field is identically zero.

molecules driven by external time-dependent fields was modeled with the help of Euler's equations [33]

$$
\mathbf{I} \dot{\boldsymbol{\Omega}}=(\mathbf{I} \boldsymbol{\Omega}) \times \boldsymbol{\Omega}+\mathbf{T},
$$

where $\mathbf{I}=\operatorname{diag}\left(I_{a}, I_{b}, I_{c}\right)$ is the moment of inertia tensor $\left(I_{a} \leq I_{b} \leq I_{c}\right), \Omega=\left(\Omega_{a}, \Omega_{b}, \Omega_{c}\right)$ is the angular velocity, and $\mathbf{T}=\left(T_{a}, T_{b}, T_{c}\right)$ is the external torque expressed in the molecular frame. The latter is given by $\mathbf{T}=\boldsymbol{\mu} \times Q^{T} \mathbf{E}$, where $\mathbf{E}$ is the external electric field defined in the laboratory frame, and $Q(t)$ is a $4 \times 3$ matrix composed of the elements of a quaternion $[34,35]$. A quaternion is defined as a quadruplet of real numbers, $q=\left(q_{0}, q_{1}, q_{2}, q_{3}\right)$ and it has a simple equation of motion $\dot{q}=q \Omega / 2$, where $\Omega=(0, \Omega)$ is a pure quaternion and the quaternions multiplication law is implied $[34,35]$. To simulate the behavior of a thermal ensemble, we use the Monte Carlo approach. For each molecule, we numerically solve the system of Euler [Eq. (1)] and quaternion equations of motion using the standard fourth order Runge-Kutta algorithm. Our ensemble consists of $N=10^{7}$ sample molecules, which were initially isotropically oriented. The initial uniform random quaternions were generated using the recipe from Ref. [36]. Initial molecular angular velocities were generated according to the Boltzmann distribution $f\left(\Omega_{i}\right) \propto \exp \left[-I_{i} \Omega_{i}^{2} /\left(2 k_{B} T\right)\right], i=a, b, c$, where $T$ is the rotational temperature and $k_{B}$ is the Boltzmann constant.

Simulation results. - In this work, we used methyl chloride $\left(\mathrm{CH}_{3} \mathrm{Cl}\right)$ and propylene oxide $\left(\mathrm{PPO}, \mathrm{CH}_{3} \mathrm{CHCH}_{2} \mathrm{O}\right)$ molecules, as typical examples of symmetric- and asymmetric-top molecules, respectively. Moments of inertia and components of the dipole moment of these molecules are provided in Supplementary Note 2.

Figure 2 shows time-dependent ensemble-averaged projection of the dipole moment, $\left\langle\mu_{Z}\right\rangle(t)$ on the laboratory $Z$ axis, along which the $\mathrm{THz}$ pulse is polarized. The angle brackets $\langle\cdot\rangle$ denote thermal averaging over the initial molecular state. The initial temperature is set to $T=5$ $K$. The two other averaged projections of the molecular dipole, $\left\langle\mu_{X}\right\rangle(t)$ and $\left\langle\mu_{Y}\right\rangle(t)$ are zero, due to the axial symmetry of the system. On the short time scale (first
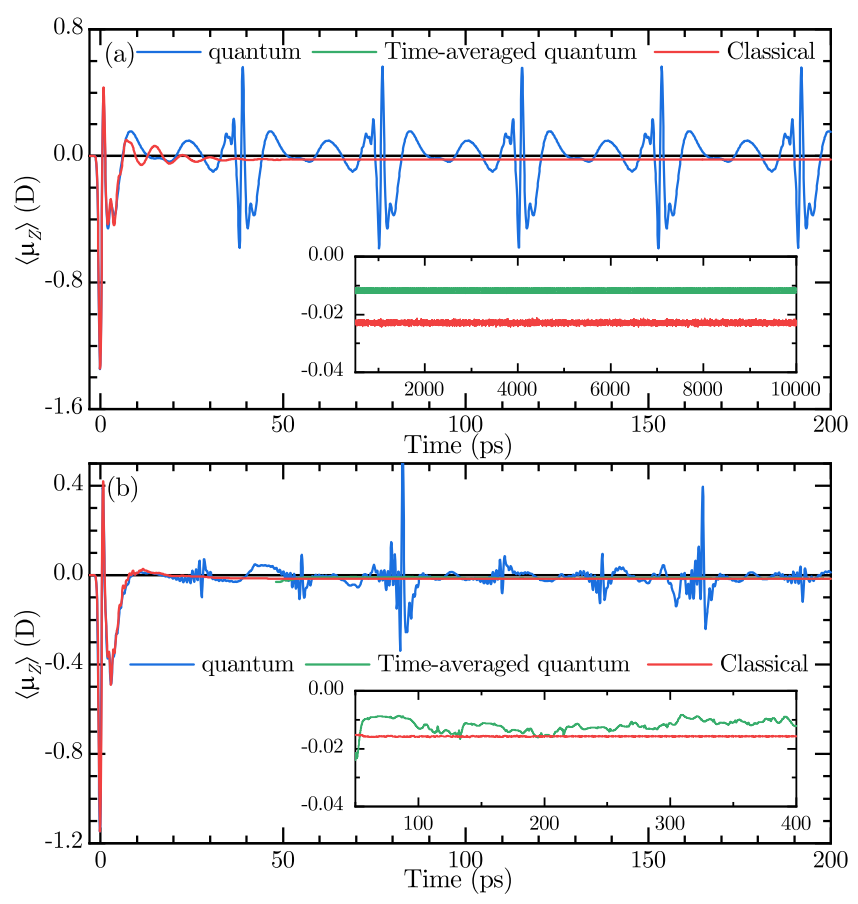

Figure 2. Ensemble averaged $Z$-projection of the dipole moment in the laboratory frame, $\left\langle\mu_{Z}\right\rangle$ as a function of time for (a) $\mathrm{CH}_{3} \mathrm{Cl}$ and (b) PPO molecules. The results of the quantum and classical simulations are shown in blue and red lines, respectively. The green curve represents the sliding time average defined by $\overline{\left\langle\mu_{Z}\right\rangle(t)}=(\Delta t)^{-1} \int_{t-\Delta t / 2}^{t+\Delta t / 2} \mathrm{~d} t^{\prime}\left\langle\mu_{Z}\right\rangle\left(t^{\prime}\right)$, where (a) $\Delta t=1000 \mathrm{ps}$ and (b) $\Delta t=100 \mathrm{ps}$, respectively. The insets show amagnified portion of the signals.

$\approx 10 \mathrm{ps}$ ), the quantum results are in good agreement with the classical ones. Both symmetric- [Fig. 2(a)] and asymmetric-top [Fig. 2(b)] systems immediately respond to the $\mathrm{THz}$ pulse by a transient dipole signal. However, long after the pulse, the orientation does not completely disappear and a long-lasting persistent dipole signal remains (see insets of Fig. 2). This remarkable behaviour is the main result of our work.

In the classical limit, the long lasting orientation of symmetric- and asymmetric-top molecules remains constant indefinitely (until the collision destroy the orientation). Quantum simulations, on the other hand, exhibit characteristic quasi periodic beats [21] (quantum revivals). However, a moving window average of the quantum results (see captions of Fig. 2) remains constant as the classically predicted orientation behavior. As can be seen in caption of Fig. 2(a), the time-averaged quantum signal is constant in the case of symmetric-top molecules. In the case of asymmetric-top molecules [Fig. 2(b)] the time-averaged signal is long-lasting as well (thousands of ps in the present case). On the even longer timescale, the absolute value of orientation slowly decreases and eventually changes its sign (not shown). The difference between symmetric- and asymmetric-top molecules 
(a) $\uparrow z$

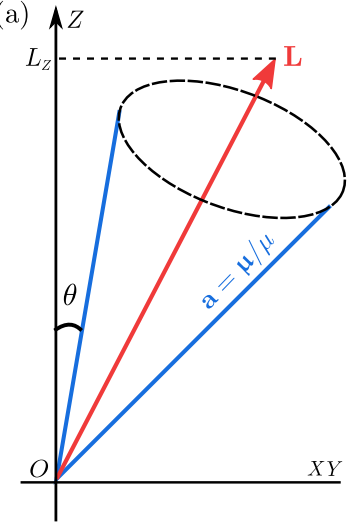

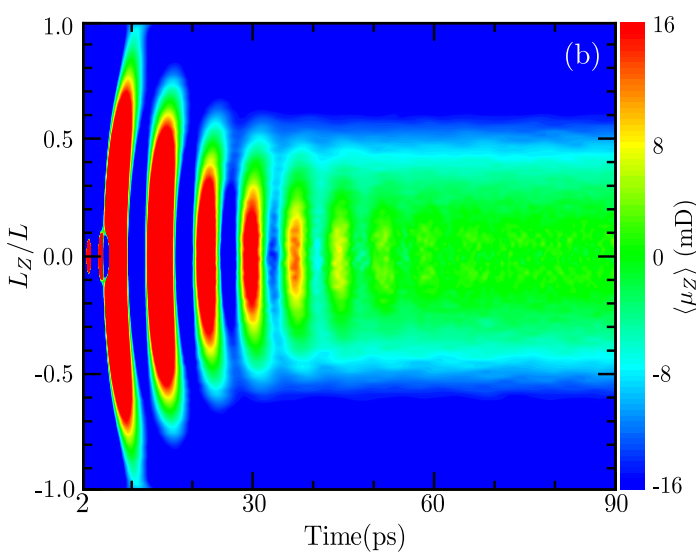

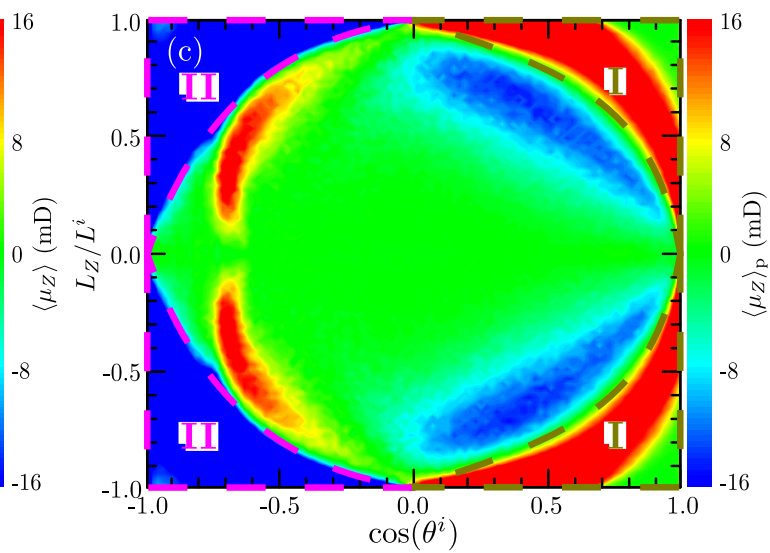

Figure 3. Thermal ensemble of classical symmetric-top molecules excited by $Z$-polarized THz pulse. (a) Illustration of the precession motion of the molecular symmetry axis a (in blue) about the angular momentum vector $\mathbf{L}$ (in red), $\theta$ is the polar angle between $\mathbf{a}(\boldsymbol{\mu})$ and the $Z$-axis. (b) The dipole signal $\left\langle\mu_{Z}\right\rangle$ as a function of time and $L_{Z} / L$. (c) Permanent value of the dipole signal $\left\langle\mu_{Z}\right\rangle_{\mathrm{p}}$ after the pulse as a function of the initial conditions before the pulse: $\cos \theta^{i}$ and $L_{Z} / L^{i}$. Two regions, I and II, are marked in (c). The color scales in (b) and (c) are in the units of millidebye (mD).

stems from the fact that quantum-mechanical states of the latter are non-degenerate and have different parity [30]. As a consequence, any state that is internally oriented at some point in time cannot be an eigenstate and will oscillate between being oriented and anti-oriented, an effect known as dynamical tunneling [37].

The results presented in Fig. 2(a) showing a series of identical revivals are valid within the rigid rotor approximation. Two additional factors should be mentioned: centrifugal distortion and radiation emission due to fast changes in the dipole moment. The centrifugal distortion leads to eventual decay of the periodic quantum revival peaks $[10,38]$ due to the dephasing of the rotational states. However, dipole signal averaged over multiple revival periods is hardly affected (see Supplementary Note $3)$. Fast variation of the dipole signal during each revival event may lead to radiative emission and gradual reduction of the rotational energy [10, 38]. However, in the case of a rarified molecular gas considered here, the estimated relative energy loss during a single revival is negligible.

Recently, a related phenomenon of persistent orientation was reported [39, 40], where twisted-polarization laser pulses were applied to the chiral molecules. Here, on the other hand, we use a single unshaped $\mathrm{THz}$ pulse, chirality is not required and the underlying mechanism is different, as is discussed in the next section.

Qualitative discussion.-To understand the origin of the long lasting orientation shown in Fig. 2, we first analyze the case of an ensemble of classical symmetrictop molecules excited by a $Z$ polarized $\mathrm{THz}$ pulse. The motion of free, prolate for definiteness, symmetric-top is defined by a simple vectorial differential equation $\dot{\mathbf{a}}=(\mathbf{L} / I) \times \mathbf{a}$, where $\mathbf{a}$ is a unit vector along the molecular axis of symmetry, $I$ is the moment of inertia
$\left(I_{a}<I_{b}=I_{c} \equiv I\right)$, and $\mathbf{L}$ is the vector of angular momentum. Explicit solution of this equation is provided in Supplementary Note 4. Figure 3(a) shows the general motion of a symmetric-top, namely precession of the unit vector a about the vector $\mathbf{L}$ at rate $L / I$, where $L$ is the magnitude of the angular momentum. The timeaveraged projection of the dipole on the $Z$ axis is given by

$$
\overline{\mu_{Z}}=\mu \lim _{\tau \rightarrow \infty} \frac{1}{\tau} \int_{0}^{\tau} \mathbf{a}(t) \cdot \mathbf{Z} \mathrm{d} t=\mu \frac{L_{Z} L_{a}}{L^{2}},
$$

where $\mu$ is the magnitude of the molecular dipole moment. The solution $\mathbf{a}(t)$ describes the field-free stage of the motion, therefore $L_{a}$ and $L$ in the above equation are taken at the end of the $\mathrm{THz}$ pulse. Notice that the projection of the angular momentum on the axis of the pulse, $L_{Z}$ is a constant of motion. The projection, $L_{a}$ of the angular momentum on the molecular symmetry axis can be expressed in terms of $L_{X}, L_{Y}, L_{Z}$, and the Euler angles [30]: $L_{a}=L_{X} \sin \theta \cos \phi+L_{Y} \sin \theta \sin \phi+L_{Z} \cos \theta$.

Thus, we have $\overline{\mu_{Z}} / \mu=\left(L_{Z} / L\right)^{2} \cos \theta+$ $\left(L_{X} L_{Z} / L^{2}\right) \sin \theta \cos \phi+\left(L_{Y} L_{Z} / L^{2}\right) \sin \theta \sin \phi$. Denoting $\cos \theta=x$ and $L_{Z} / L=y=\cos \theta_{1}$, the ensemble averaged dipole moment is given by

$$
\overline{\left\langle\mu_{Z}\right\rangle} \propto \mu \int_{-1}^{1} x P_{0}(x) \mathrm{d} x,
$$

where $P_{0} \propto \int y^{2} L^{2} P_{1}(x, y, L) \mathrm{d} L \mathrm{~d} y$ and $P_{1}(x, y, L)$ is the joint probability distribution of $\cos \theta, \cos \theta_{1}$ and $L$ at the end of the THz pulse. Terms proportional to $\sin \phi$ and $\cos \phi$ in the expression for $\overline{\mu_{Z}} / \mu$ do not contribute to the average, because a $\mathrm{THz}$ pulse does not affect a uniform distribution of the angle $\phi$. For initial temperature 
$T=0 \mathrm{~K}, \overline{\mu_{Z}}$ vanishes because $L_{Z} \equiv 0$ before and after the $\mathrm{THz}$ pulse. Finally, in the limit of a pulse of vanishing duration $(\kappa \rightarrow \infty$, see Fig. 1), the permanent orientation tends to zero because $P_{0}$ keeps the initial symmetric distribution just after the pulse. As an illustration, the joint probability distribution $P_{1}$ can be written down explicitly for a model system of thermalized symmetric-top molecules $\left(I / I_{a}=w>1\right)$ subject to a constant dc field of amplitude $E_{0}$ which is then abruptly switched off. In this case, $P_{1}$ is separable and is given by $P_{1}(x, y, l) \propto$ $f(x) g(y, l)=\exp (\epsilon x) \exp \left\{-l^{2}\left[1+y^{2}(w-1)\right]\right\}$, where $\epsilon=\mu E_{0} / k_{B} T$ and $l=L / \sqrt{2 I k_{B} T}$ (see Supplementary Note 5). Clearly, $\overline{\left\langle\mu_{Z}\right\rangle} \neq 0$, because $\exp (\epsilon x)$ is not symmetric in the interval $x \in[-1,1]$ for $\epsilon>0$.

To explore the long lasting orientation and to better understand its physical origin, we consider the underlying dynamics in more detail. After the end of the pulse, at $t>2$ ps (see Fig. 1), the ratio $L_{Z} / L$ is constant. Figure $3(\mathrm{~b})$ shows the ensemble averaged dipole moment, $\left\langle\mu_{Z}\right\rangle$ in a three dimensional color-coded plot, as a function of time and the (constant) ratio $L_{Z} / L$. As can be seen in the figure, right after the pulse $(t=2 \mathrm{ps})$ approximately all the molecules have a negative $Z$ projection of their dipoles. Moreover, Fig. 3(b) shows that, at long times, molecules with $L_{Z} \simeq \pm L$ (upper and lower blue bands) contribute to the overall negative dipole signal, while for molecules with $\left|L_{Z} / L\right| \ll 1$ (central portion of the figure), the time-averaged dipole signal tends to zero. In the absence of external fields, the molecules with angular momentum along $\pm Z$ axis, and the symmetry axis initially directed along $-Z$, will continue to precess around the $-Z$ direction forever. In contrast, the contribution of molecules with angular momentum lying in the $X Y$ plane $\left(\left|L_{Z} / L\right| \ll 1\right)$ averages to zero and they do not contribute to the long lasting orientation. In the special case of initial temperature of $T=0 \mathrm{~K}, L_{Z}$ remains identically zero after the pulse $\left(L_{Z}\right.$ is conserved), thus forbidding the permanent orientation.

The physical origin of the permanent (negative) orientation observed in Fig. 3(b) can be further analysed by considering it as a function of the initial orientation factor, $\cos \theta^{i}$ and the value of $L_{Z} / L^{i}$ before the pulse. Figure 3(c) shows the value of $\left\langle\mu_{Z}\right\rangle$ taken at times after 100 ps [see Fig. 2(a)], denoted as $\left\langle\mu_{Z}\right\rangle_{\mathrm{p}}$, as a function of the initial conditions. As a reminder, $\theta^{i}$ is the initial angle between the dipole moment and the $Z$-axis [see Fig. $3(\mathrm{a})]$. The figure shows that molecules with $|\cos \theta| \simeq 1$ and $L_{Z} / L^{i}= \pm 1$ (regions denoted by I and II, blue and red in the figure) maintain the nonzero permanent orientation, while the orientation of molecules in other regions tends to zero. To understand the reason for this, we consider molecules in each region separately and follow their time evolution in the presence of the $\mathrm{THz}$ pulse.

Initially, molecules in region I precess about the $+Z$ direction, because $L_{Z} \simeq \pm L^{i}$ and $\cos \theta^{i} \simeq 1$. During the first phase of the THz pulse, when the field points
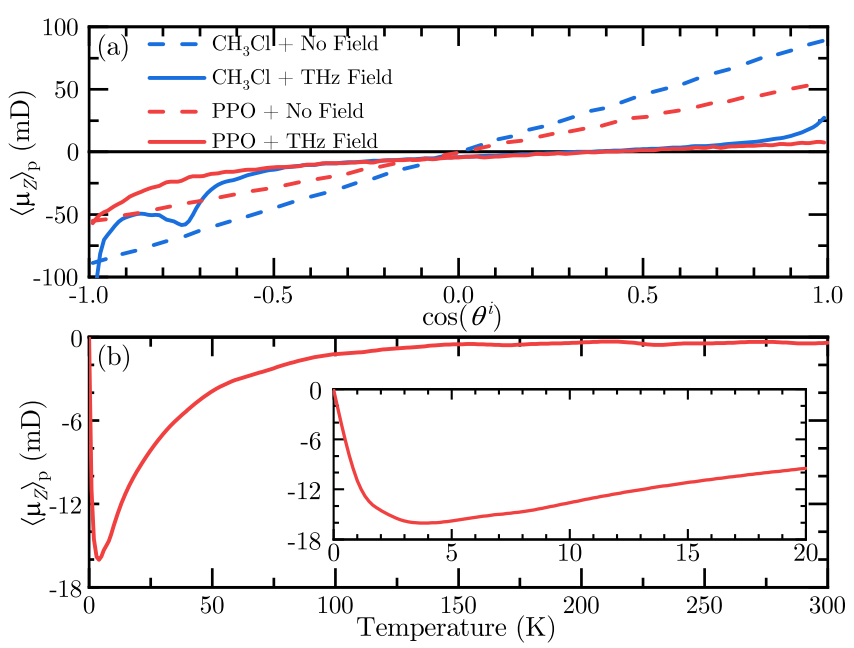

Figure 4. (a) Classically calculated permanent values of the dipole signals $\left\langle\mu_{Z}\right\rangle_{\mathrm{p}}$ as a function of initial angular position $\cos \theta^{i}$ for four cases: $\mathrm{CH}_{3} \mathrm{Cl}$ without field (dashed blue), $\mathrm{CH}_{3} \mathrm{Cl}$ and $\mathrm{THz}$ field (solid blue), PPO without field (dashed red), and PPO and THz field (solid red). (b) Classically calculated permanent values of the dipole signal $\left\langle\mu_{Z}\right\rangle_{\mathrm{p}}$ as a function of temperature for PPO molecule.

along $-Z$ (see Fig. 1), it tends to flip the molecular dipoles. In contrast, during the second phase, when the field is along $+Z$ (see Fig. 1), it pushes the dipoles towards $+Z$, thus facilitating the permanent orientation. Molecules in region II, which initially precess about $-Z$ $\left(L_{Z} \simeq \pm L^{i}, \cos \theta^{i} \simeq-1\right)$ respond oppositely as compared to the molecules in region I. For the chosen parameters of the THz pulse (see Fig. 1), there is an imbalance between these two groups resulting in the excess of molecules precessing about $-Z$ axis. This leads to the overall negative permanent orientation.

The qualitative mechanism described above for the case of symmetric-top molecules is essentially the same for asymmetric-top molecules as well. In order to establish the similarity between the symmetric- and asymmetric-top molecules, Fig. 4(a) depicts the permanent values of the dipole signals $\left\langle\mu_{Z}\right\rangle_{\mathrm{p}}$ as a function of initial orientation quantified by $\cos \theta^{i}$ for the previously considered methyl chloride and propylene oxide molecules. In both cases, the $\mathrm{THz}$ pulse preferentially supports the molecular precession about the $-Z$ direction, as opposed to $+Z$, so that the overall long-time dipole value is negative.

It was pointed out earlier that at $T=0 \mathrm{~K}$ permanent orientation is impossible. Also, as $T \rightarrow \infty$ the orientation disappears, as well. This non-monotonous temperature dependence leads to the existence of an optimal initial temperature that will provide the maximal (in absolute value) permanent orientation. Indeed, Fig. 4(b) depicts the temperature dependence of the permanent dipole signal, calculated classically. As can be seen in the figure, 
for the pulse parameters used here, the maximal persistent orientation is achieved for initial temperature of $T \approx 4 \mathrm{~K}$.

Summary.-We have theoretically demonstrated a new phenomenon of persistent orientation of symmetricand asymmetric-top molecules excited by a single $\mathrm{THz}$ pulse. The orientation was shown to persist long after the end of the pulse for both types of molecules. Analysis of the relatively simple special case of symmetric-top molecules reveals the underlying classical mechanism and provides a detailed understanding. The mechanism is general and relies on the symmetry breaking of the molecular ensemble caused by the interaction with the external field. Similar mechanism may lead to persistent orientation effect in the cases of symmetricand asymmetric-tops excited by two-color laser fields [41-45]. The fast transient dipole signal and its long-time persistent component may be directly measured with the help of second (or higher order) harmonic generation, which is sensitive to the lack of inversion symmetry [44].

This work was supported by the Israel Science Foundation (Grant No. 746/15), the ISF-NSFC joint research program (Grant No. 2520/17). I.A. acknowledges support as the Patricia Elman Bildner Professorial Chair. This research was made possible in part by the historic generosity of the Harold Perlman Family.

* L. X. and I. T. contributed equally to this work.

† ilya.averbukh@weizmann.ac.il

[1] H. Stapelfeldt and T. Seideman, Colloquium: Aligning molecules with strong laser pulses, Rev. Mod. Phys. 75, 543 (2003).

[2] Y. Ohshima and H. Hasegawa, Coherent rotational excitation by intense nonresonant laser fields, Int. Rev. Phys. Chem. 29, 619 (2010).

[3] S. Fleischer, Y. Khodorkovsky, E. Gershnabel, Y. Prior, and I. S. Averbukh, Molecular alignment induced by ultrashort laser pulses and its impact on molecular motion, Isr. J. Chem. 52, 414 (2012).

[4] M. Lemeshko, R. V. Krems, J. M. Doyle, and S. Kais, Manipulation of molecules with electromagnetic fields, Mol. Phys. 111, 1648 (2013).

[5] C. P. Koch, M. Lemeshko, and D. Sugny, Quantum control of molecular rotation, Rev. Mod. Phys. 91, 035005 (2019).

[6] K.-L. Yeh, M. C. Hoffmann, J. Hebling, and K. A. Nelson, Generation of $10 \mu \mathrm{J}$ ultrashort terahertz pulses by optical rectification, Appl. Phys. Lett. 90, 171121 (2007).

[7] S. Fleischer, Y. Zhou, R. W. Field, and K. A. Nelson, Molecular orientation and alignment by intense singlecycle THz pulses, Phys. Rev. Lett. 107, 163603 (2011).

[8] K. Kitano, N. Ishii, N. Kanda, Y. Matsumoto, T. Kanai, M. Kuwata-Gonokami, and J. Itatani, Orientation of jetcooled polar molecules with an intense single-cycle $\mathrm{THz}$ pulse, Phys. Rev. A 88, 061405 (2013).

[9] K. N. Egodapitiya, S. Li, and R. R. Jones, Terahertz- induced field-free orientation of rotationally excited molecules, Phys. Rev. Lett. 112, 103002 (2014).

[10] P. Babilotte, K. Hamraoui, F. Billard, E. Hertz, B. Lavorel, O. Faucher, and D. Sugny, Observation of the field-free orientation of a symmetric-top molecule by terahertz laser pulses at high temperature, Phys. Rev. A 94, 043403 (2016).

[11] R. Damari, S. Kallush, and S. Fleischer, Rotational control of asymmetric molecules: Dipoleversus polarizability-driven rotational dynamics, Phys. Rev. Lett. 117, 103001 (2016).

[12] O. Atabek, C. M. Dion, and A. B. H. Yedder, Evolutionary algorithms for the optimal laser control of molecular orientation, J. Phys. B 36, 4667 (2003).

[13] D. Daems, S. Guérin, D. Sugny, and H. R. Jauslin, Efficient and long-lived field-free orientation of molecules by a single hybrid short pulse, Phys. Rev. Lett. 94, 153003 (2005).

[14] E. Gershnabel, I. S. Averbukh, and R. J. Gordon, Orientation of molecules via laser-induced antialignment, Phys. Rev. A 73, 061401 (2006).

[15] K. Kitano, N. Ishii, and J. Itatani, High degree of molecular orientation by a combination of $\mathrm{THz}$ and femtosecond laser pulses, Phys. Rev. A 84, 053408 (2011).

[16] C.-C. Shu and N. E. Henriksen, Field-free molecular orientation induced by single-cycle $\mathrm{THz}$ pulses: The role of resonance and quantum interference, Phys. Rev. A 87, 013408 (2013).

[17] M. Yoshida and Y. Ohtsuki, Control of molecular orientation with combined near-single-cycle $\mathrm{THz}$ and optimally designed non-resonant laser pulses: Carrier-envelope phase effects, Chem. Phys. Lett. 633, 169 (2015).

[18] M. Mirahmadi, B. Schmidt, M. Karra, and B. Friedrich, Dynamics of polar polarizable rotors acted upon by unipolar electromagnetic pulses: From the sudden to the adiabatic regime, J. Chem. Phys. 149, 174109 (2018).

[19] C. A. Arango and G. S. Ezra, Classical mechanics of dipolar asymmetric top molecules in collinear static electric and nonresonant linearly polarized laser fields: Energy-momentum diagrams, bifurcations and accessible configuration space, Int. J. Bifurc. Chaos Appl. Sci. Eng. 18, 1127 (2008).

[20] K. Schatz, B. Friedrich, S. Becker, and B. Schmidt, Symmetric tops in combined electric fields: Conditional quasisolvability via the quantum Hamilton-Jacobi theory, Phys. Rev. A 97, 053417 (2018).

[21] P. M. Felker, Rotational coherence spectroscopy: Studies of the geometries of large gas-phase species by picosecond time-domain methods, J. Phys. Chem. 96, 7844 (1992).

[22] I. S. Averbukh and N. F. Perelman, Fractional revivals: Universality in the long-term evolution of quantum wave packets beyond the correspondence principle dynamics, Phys. Lett. A 139, 449 (1989).

[23] R. W. Robinett, Quantum wave packet revivals, Phys. Rep 392, 1 (2004).

[24] R. V. Krems, Molecules in Electromagnetic Fields: From Ultracold Physics to Controlled Chemistry (Wiley, 2018).

[25] L. H. Coudert, Optimal orientation of an asymmetric top molecule with terahertz pulses, J. Chem. Phys. 146, 024303 (2017).

[26] M. Clerici, M. Peccianti, B. E. Schmidt, L. Caspani, M. Shalaby, M. Giguère, A. Lotti, A. Couairon, F. Légaré, T. Ozaki, D. Faccio, and R. Morandotti, Wavelength scaling of terahertz generation by gas ionization, 
Phys. Rev. Lett. 110, 253901 (2013).

[27] T. I. Oh, Y. J. Yoo, Y. S. You, and K. Y. Kim, Generation of strong terahertz fields exceeding $8 \mathrm{MV} / \mathrm{cm}$ at $1 \mathrm{kHz}$ and real-time beam profiling, Appl. Phys. Lett. 105, 041103 (2014).

[28] C. Vicario, A. V. Ovchinnikov, S. I. Ashitkov, M. B. Agranat, V. E. Fortov, and C. P. Hauri, Generation of 0.9-mJ THz pulses in DSTMS pumped by a Cr: $\mathrm{Mg}_{2} \mathrm{SiO}_{4}$ laser, Opt. Lett. 39, 6632 (2014).

[29] M. Shalaby and C. P. Hauri, Demonstration of a lowfrequency three-dimensional terahertz bullet with extreme brightness, Nat. Commun. 6, 5976 (2015).

[30] R. N. Zare, Angular momentum: understanding spatial aspects in chemistry and physics (Wiley, New York, 1988).

[31] R. B. Sidje, Expokit: A software package for computing matrix exponentials, ACM Trans. Math. Softw. 24, 130 (1998).

[32] R. S. McDowell, Rotational partition functions for symmetric-top molecules, J. Chem. Phys. 93, 2801 (1990).

[33] H. Goldstein, C. Poole, and J. Safko, Classical Mechanics (Addison Wesley, San Francisco, CA, 2002).

[34] E. A. Coutsias and L. Romero, The quaternions with an application to rigid body dynamics, Sandia Technical Report, SAND2004-0153 (2004).

[35] J. B. Kuipers, Quaternions and Rotation Sequences: A Primer with Applications to Orbits, Aerospace and Virtual Reality (Princeton University Press, Princeton, N.J., 1999).

[36] S. M. LaValle, Planning Algorithms (Cambridge University Press, New York, 2006).

[37] S. Keshavamurthy and P. Schlagheck, Dynamical tunneling: theory and experiment (CRC Press, Boca Raton, 2011).
[38] R. Damari, D. Rosenberg, and S. Fleischer, Coherent radiative decay of molecular rotations: A comparative study of terahertz-oriented versus optically aligned molecular ensembles, Phys. Rev. Lett. 119, 033002 (2017).

[39] I. Tutunnikov, J. Floß, E. Gershnabel, P. Brumer, and I. S. Averbukh, Laser-induced persistent orientation of chiral molecules, Phys. Rev. A 100, 043406 (2019).

[40] I. Tutunnikov, J. Floß, E. Gershnabel, P. Brumer, I. S. Averbukh, A. A. Milner, and V. Milner, Observation of persistent orientation of chiral molecules by a laser field with twisted polarization, Phys. Rev. A 101, 021403 (2020).

[41] S. De, I. Znakovskaya, D. Ray, F. Anis, N. G. Johnson, I. A. Bocharova, M. Magrakvelidze, B. D. Esry, C. L. Cocke, I. V. Litvinyuk, and M. F. Kling, Field-free orientation of $\mathrm{CO}$ molecules by femtosecond two-color laser fields, Phys. Rev. Lett. 103, 153002 (2009).

[42] K. Oda, M. Hita, S. Minemoto, and H. Sakai, All-optical molecular orientation, Phys. Rev. Lett. 104, 213901 (2010).

[43] J. Wu and H. Zeng, Field-free molecular orientation control by two ultrashort dual-color laser pulses, Phys. Rev. A 81, 053401 (2010).

[44] E. Frumker, C. T. Hebeisen, N. Kajumba, J. B. Bertrand, H. J. Wörner, M. Spanner, D. M. Villeneuve, A. Naumov, and P. B. Corkum, Oriented rotational wavepacket dynamics studies via high harmonic generation, Phys. Rev. Lett. 109, 113901 (2012).

[45] K. Lin, I. Tutunnikov, J. Qiang, J. Ma, Q. Song, Q. Ji, W. Zhang, H. Li, F. Sun, X. Gong, H. Li, P. Lu, H. Zeng, Y. Prior, I. S. Averbukh, and J. Wu, All-optical field-free three-dimensional orientation of asymmetrictop molecules, Nat. Commun. 9, 5134 (2018). 